\title{
A CHOQUET BOUNDARY FOR THE PRODUCT OF TWO COMPACT SPACES
}

\author{
MARVIN W. GROSSMAN ${ }^{1}$
}

Let $X$ be a compact Hausdorff space and $C(X)$ the Banach space of all real-valued continuous functions on $X$ under the sup norm. If $H$ is a linear subspace of $C(X)$ which separates the points of $X$ and contains the constant functions, then it is well known that there exists a smallest closed subset of $X$, called the Silov boundary of $X$ relative to $H$ and denoted by $\partial_{H} X$, with the property that each $h \in H$ attains its maximum value on $\partial_{H} X$. A point $x \in X$ is called an $H$-extremal point if the only positive linear functional $u$ on $C(X)$ such that $u(h)=h(x)$ for all $h \in H$ is the evaluation functional $\phi_{x}$ where $\phi_{x}(f)$ $=f(x)$ for all $f \in C(X)$. The set of $H$-extremal points of $X$ is called the Choquet boundary of $X$ relative to $H$ and will be denoted by $\nabla_{H} X$. H. Bauer has shown $[1,2.1]$ that the Choquet boundary of $X$ relative to $H$ is nonempty and the Silov boundary of $X$ relative to $H$ is equal to the closure of the Choquet boundary.

Bauer has also introduced (see [1]) an abstract Dirichlet problem for the above setting. If $S \supset \partial_{H} X$ is closed and $x \in X$, then $M_{x}^{S}$ denotes the set of positive linear functionals $u$ on $C(S)$ such that $u(h \mid S)$ $=h(x)$ for all $h \in H . M_{x}^{S}$ is always nonempty. The measures in $M_{x}^{\partial_{H} X}$ are called the $H$-harmonic measures belonging to $x$. A function $f$ in $C(X)$ is said to be $H$-harmonic if for every $x \in X$ and every $u \in M_{x}^{X}$ we have $u(f)=f(x)$. The set of $H$-harmonic functions is denoted by $\hat{H}$. The Dirichlet problem is said to be solvable for $X$ relative to $H$ if $\hat{H} \mid \partial_{H} X=C\left(\partial_{H} X\right)$. The Dirichlet problem is solvable if and only if to each $x$ in $X$ belongs exactly one $H$-harmonic measure (see [ 1 , Satz 9]).

Let $X_{1}, X_{2}$ be compact Hausdorff spaces and $H_{1}, H_{2}$ separating linear subspaces of $C\left(X_{1}\right), C\left(X_{2}\right)$ respectively, which contain the constant functions. Equipped with the usual product topology, the cartesian product $X_{1} \times X_{2}$ is a compact Hausdorff space. In this paper we show that a subfamily $H_{1}+H_{2}$ of $C\left(X_{1} \times X_{2}\right)$ can be defined, in a natural way, for which $\nabla_{H_{1}+H_{2}} X_{1} \times X_{2}$ exists and $\nabla_{H_{1}+H_{2}} X_{1} \times X_{2}$ $=\nabla_{H_{1}} X_{1} \times \nabla_{H_{2}} X_{2}$. We then give two theorems on the relation of the

Received by the editors July 7, 1964.

${ }^{1}$ The material in this paper appears in the author's doctoral dissertation at the University of Illinois. The research was supported in part by the National Science Foundation under Grant G-19869. 
solvability of the Dirichlet problem for $X_{1}$ and $X_{2}$ to the solvability of the Dirichlet problem for $X_{1} \times X_{2}$.

If $H$ is a separating linear subspace of $C(X)$ which contains the constant functions, then $H^{*}$ will denote the conjugate space of $H$ where $H$ is equipped with the sup norm and $U_{H}$ will denote the positive face of the unit sphere of $H^{*}$, i.e., the set of positive linear functionals $u$ on $H$ such that $u(1)=1$. We will make use of Bishop and de Leeuw's characterization of the Choquet boundary in terms of geometric extreme points. Namely, $x \in \nabla_{H} X$ if and only if $\phi_{x}^{H}$ is an extreme point of $U_{H}$ where $\phi_{x}^{H}(h)=h(x)$ for all $h \in H$ (see [1, Hilfssatz 8] or [2, Lemma 4.3]).

Definition. If $h_{1} \in H_{1}$ and $h_{2} \in H_{2}$, then $\left[h_{1}+h_{2}\right]$ will denote the function defined on $X_{1} \times X_{2}$ as follows:

$$
\left[h_{1}+h_{2}\right](x, y)=h_{1}(x)+h_{2}(y) \text { for all }(x, y) \in X_{1} \times X_{2} \text {. }
$$

We set $H_{1}+H_{2}=\left\{\left[h_{1}+h_{2}\right]: h_{1} \in H_{1}, h_{2} \in H_{2}\right\}$.

Let $(x, y),\left(\dot{x}_{0}, y_{0}\right) \in X_{1} \times X_{2}$ and $\left[h_{1}+h_{2}\right] \in H_{1}+H_{2}$. From the inequality $\left|h_{1}(x)+h_{2}(y)-h_{1}\left(x_{0}\right)-h_{2}\left(y_{0}\right)\right| \leqq\left|h_{1}(x)-h_{1}\left(x_{0}\right)\right|$ $+\left|h_{2}(y)-h_{2}\left(y_{0}\right)\right|$ it is evident that $H_{1}+H_{2} \subset C\left(X_{1} \times X_{2}\right)$. It is clear from the definition that $H_{1}+H_{2}$ is a linear subspace containing the constant functions. Let $(x, y) \neq\left(x^{\prime}, y^{\prime}\right)$ be elements of $X_{1} \times X_{2}$. For definiteness we may assume $x \neq x^{\prime}$. Since $H_{1}$ separates the points of $X_{1}$, there exists $h_{1} \in H_{1}$ such that $h_{1}(x) \neq h_{1}\left(x^{\prime}\right)$. Then $\left[h_{1}+0\right](x, y)$ $\neq\left[h_{1}+0\right]\left(x^{\prime}, y^{\prime}\right)$. Thus, $H_{1}+H_{2}$ separates the points of $X_{1} \times X_{2}$. It follows that the Silov boundary $\partial_{H_{1}+H_{2}} X_{1} \times X_{2}$ exists and $\mathrm{Cl}\left(\nabla_{H_{1}+H_{2}} X_{1} \times X_{2}\right)=\partial_{H_{1}+H_{2}} X_{1} \times X_{2}$ where $\nabla_{H_{1}+H_{2}} X_{1} \times X_{2}$ is the Choquet boundary of $X_{1} \times X_{2}$ relative to $H_{1}+H_{2}$ and $\mathrm{Cl}$ denotes closure.

Theorem 1. $\partial_{H_{1}} X_{1} \times \partial_{H_{2}} X_{2}=\partial_{H_{1}+H_{2}} X_{1} \times X_{2}$.

Proof. Let $\left[h_{1}+h_{2}\right] \in H_{1}+H_{2}$. From the equality $\sup _{x_{1} \times x_{2}}\left[h_{1}+h_{2}\right]$ $=\sup _{X_{1}} h_{1}+\sup _{X_{2}} h_{2}$ and the fact that $\partial_{H_{1}} X_{1} \times \partial_{H_{2}} X_{2}$ is closed in $X_{1} \times X_{2}$, it follows immediately that $\partial_{H_{1}+H_{2}} X_{1} \times X_{2} \subset \partial_{H_{1}} X_{1} \times \partial_{H_{2}} X_{2}$.

Now let $\left(x_{0}, y_{0}\right) \in \partial_{H_{1}} X_{1} \times \partial_{H_{2}} X_{2}$. Let $U_{x_{0}}$ and $V_{y_{0}}$ be neighborhoods of $x_{0}$ and $y_{0}$ respectively. Since $x_{0} \in \partial_{H_{1}} X_{1}$ and $y_{0} \in \partial_{H_{2}} X_{2}$, there exist $h_{1} \in H_{1}$ and $h_{2} \in H_{2}$ such that $\left\{x: h_{1}(x)=\sup _{x_{1}} h_{1}\right\} \subset U_{x_{0}}$ and $\left\{y: h_{2}(y)\right.$ $\left.=\sup _{x_{2}} h_{2}\right\} \subset V_{y_{0}}$. If $\left(x^{\prime}, y^{\prime}\right) \notin U_{x_{0}} \times V_{y_{0}}$, then either $h_{1}\left(x^{\prime}\right)<\sup _{x_{1}} h_{1}$ or $h_{2}\left(y^{\prime}\right)<\sup _{X_{2}} h_{2}$. Thus

$$
\begin{aligned}
{\left[h_{1}+h_{2}\right]\left(x^{\prime}, y^{\prime}\right) } & =h_{1}\left(x^{\prime}\right)+h_{2}\left(y^{\prime}\right)<\sup _{x_{1}} h_{1}+\sup _{x_{2}} h_{2} \\
& =\sup _{X_{1} \times X_{2}}\left[h_{1}+h_{2}\right] .
\end{aligned}
$$

Thereby, $\left\{(x, y):\left[h_{1}+h_{2}\right](x, y)=\sup _{x_{1} \times x_{2}}\left[h_{1}+h_{2}\right]\right\} \subset U_{x_{0}} \times V_{y_{0}}$. Now 
the subsets of $X_{1} \times X_{2}$ of the form $U_{x_{0}} \times V_{y_{0}}$ where $U_{x_{0}}$ and $V_{y_{0}}$ are neighborhoods of $x_{0}$ and $y_{0}$ respectively, constitute a base for the neighborhood system of $\left(x_{0}, y_{0}\right)$ in the product topology. Consequently, for every neighborhood $W$ of $\left(x_{0}, y_{0}\right)$ there exists $\left[h_{1}+h_{2}\right]$ in $H_{1}+H_{2}$ such that $\left\{(x, y):\left[h_{1}+h_{2}\right](x, y)=\sup _{X_{1} \times X_{2}}\left[h_{1}+h_{2}\right]\right\} \subset W$. Thus, $\left(x_{0}, y_{0}\right) \in \partial_{H_{1}+H_{2}} X_{1} \times X_{2}$ since the Ślov boundary is closed.

Theorem 2. $\nabla_{H_{1}} X_{1} \times \nabla_{H_{2}} X_{2}=\nabla_{H_{1}+H_{2}} X_{1} \times X_{2}$.

Proof. Suppose $(x, y) \notin \nabla_{H_{1}+H_{2}} X_{1} \times X_{2}$. Then $\phi_{(x, y)}^{H_{1}+H_{2}}$ is not an extreme point of the positive face of the unit sphere of $\left(H_{1}+H_{2}\right)^{*}$. Thus, there exists $u_{1}, u_{2} \in U_{H_{1}+H_{2}}$ with $u_{1} \neq u_{2}$ and $0<\lambda<1$ such that $\phi_{(x, y)}^{H_{1}+H_{2}}=\lambda u_{1}+(1-\lambda) u_{2}$. Note that if $\left[h_{1}+h_{2}\right] \in H_{1}+H_{2}$, then $\left[h_{1}+h_{2}\right]$ $=\left[h_{1}+0\right]+\left[0+h_{2}\right]$. Since $u_{1} \neq u_{2}$ there exists $\left[h_{1}+h_{2}\right] \in H_{1}+H_{2}$ such that $u_{1}\left(\left[h_{1}+h_{2}\right]\right) \neq u_{2}\left(\left[h_{1}+h_{2}\right]\right)$. But $u_{1}\left(\left[h_{1}+h_{2}\right]\right)=u_{1}\left(\left[h_{1}+0\right]\right)$ $+u_{1}\left(\left[0+h_{2}\right]\right)$ and $u_{2}\left(\left[h_{1}+h_{2}\right]\right)=u_{2}\left(\left[h_{1}+0\right]\right)+u_{2}\left(\left[0+h_{2}\right]\right)$. Thus, either $u_{1}\left(\left[h_{1}+0\right]\right) \neq u_{2}\left(\left[h_{1}+0\right]\right)$ or $u_{1}\left(\left[0+h_{2}\right]\right) \neq u_{2}\left(\left[0+h_{2}\right]\right)$. We assume for definiteness $u_{1}\left(\left[h_{1}+0\right]\right) \neq u_{2}\left(\left[h_{1}+0\right]\right)$. Define $a_{1}, a_{2}$ on $H_{1}$ as follows: $\mathfrak{u}_{1}(h)=u_{1}([h+0])$ and $\hat{u}_{2}(h)=u_{2}([h+0])$ for all $h \in H_{1}$. It is evident that $\mathfrak{n}_{1}$ and $\mathfrak{n}_{2}$ are positive linear functionals on $H_{1}$ such that $\mathfrak{a}_{i}(1)=1$ for $i=1,2$. Thus, $\mathfrak{u}_{1}, \mathfrak{u}_{2} \in U_{H_{1}}$. We have $\mathfrak{u}_{1} \neq \mathfrak{a}_{2}$ since

$$
a_{1}\left(h_{1}\right)=u_{1}\left(\left[h_{1}+0\right]\right) \neq u_{2}\left(\left[h_{1}+0\right]\right)=\hat{a}_{2}\left(h_{1}\right) .
$$

Now let $h \in H_{1}$; then

$$
\begin{aligned}
\lambda \mathfrak{u}_{1}(h)+(1-\lambda) \mathfrak{u}_{2}(h) & =\lambda u_{1}([h+0])+(1-\lambda) u_{2}([h+0]) \\
& =\phi_{(x, y)}^{H_{1}+H_{2}}([h+0])=h(x)=\phi_{x}^{H_{1}}(h) .
\end{aligned}
$$

Thus, $\phi_{x}^{H_{1}}=\lambda \hat{u}_{1}+(1-\lambda) \hat{u}_{2}$. Thus, $\phi_{x}^{H_{1}}$ is not an extreme point of $U_{H_{1}}$ and thereby $x \notin \nabla_{H_{1}} X_{1}$. The argument is similar if $u_{1}\left(\left[0+h_{2}\right]\right)$ $\neq u_{2}\left(\left[0+h_{2}\right]\right)$. We have shown: if $(x, y) \notin \nabla_{H_{1}+H_{2}} X_{1} \times X_{2}$, then either $x \notin \nabla_{H_{1}} X_{1}$ or $y \notin \nabla_{H_{2}} X_{2}$. Thus, $\left(\nabla_{H_{1}+H_{2}} X_{1} \times X_{2}\right)^{\prime} \subset\left(\nabla_{H_{1}} X_{1} \times \nabla_{H_{2}} X_{2}\right)^{\prime}$ where' indicates complement relative to $X_{1} \times X_{2}$. It follows that $\left(\nabla_{H_{1}} X_{1} \times \nabla_{H_{2}} X_{2}\right) \subset \nabla_{H_{1}+H_{2}} X_{1} \times X_{2}$.

Suppose $\left(x_{0}, y_{0}\right) \notin \nabla_{H_{1}} X_{1} \times \nabla_{H_{2}} X_{2}$. Then either $x_{0} \notin \nabla_{H_{1}} X_{1}$ or $y_{0} \notin \nabla_{H_{2}} X_{2}$. We assume for definiteness $x_{0} \notin \nabla_{H_{1}} X_{1}$. Thus, there exists $u \in M_{x_{0}}^{X_{1}}\left(H_{1}\right)$ and $g \in C\left(X_{1}\right)$ such that $u(g) \neq g\left(x_{0}\right)$. Define $\psi: C\left(X_{1} \times X_{2}\right)$ $\rightarrow C\left(X_{1}\right)$ as follows: if $\hat{f} \in C\left(X_{1} \times X_{2}\right)$, then $\psi(\hat{f})(x)=\hat{f}\left(x, y_{0}\right)$ for all $x \in X_{1}$. Clearly, $\psi(\hat{f}) \in C\left(X_{1}\right)$ and $\psi$ is a positive preserving linear map.

Now define $\mathfrak{u}$ on $C\left(X_{1} \times X_{2}\right)$ by $\hat{u}(\hat{f})=u(\psi(\hat{f}))$ for all $\hat{f} \in C\left(X_{1} \times X_{2}\right)$. Since $\boldsymbol{a}$ is the composition of two positive linear maps, it is a positive linear functional on $C\left(X_{1} \times X_{2}\right)$. Let $\left[h_{1}+h_{2}\right] \in H_{1}+H_{2}$. Since $h_{1}+h_{2}\left(y_{0}\right) \in H_{1}$ and $u \in M_{x_{0}}^{X_{1}}\left(H_{1}\right)$ we have $a\left(\left[h_{1}+h_{2}\right]\right)=u\left(\psi\left(\left[h_{1}+h_{2}\right]\right)\right)$ $=u\left(h_{1}+h_{2}\left(y_{0}\right)\right)=h_{1}\left(x_{0}\right)+h_{2}\left(y_{0}\right)=\left[h_{1}+h_{2}\right]\left(x_{0}, y_{0}\right)$. Now $u(g) \neq g\left(x_{0}\right)$. 
Define $\hat{g} \in C\left(X_{1} \times X_{2}\right)$ by $\hat{g}(x, y)=g(x)$ for all $(x, y) \in X_{1} \times X_{2}$. $\hat{g}$ is continuous since it is the composition of $g$ with a projection map. Then $\mathfrak{a}(\hat{g})=u(\psi(\hat{g}))=u(g) \neq g\left(x_{0}\right)=\hat{g}\left(x_{0}, y_{0}\right)$. Thus, $\hat{u}(\hat{g}) \neq \hat{g}\left(x_{0}, y_{0}\right)$. It follows that $\left(x_{0}, y_{0}\right) \notin \nabla_{H_{1}+H_{2}} X_{1} \times X_{2}$. We have shown that if $\left(x_{0}, y_{0}\right)$ $\notin \nabla_{H_{1}} X_{1} \times \nabla_{H_{2}} X_{2}$, then $\left(x_{0}, y_{0}\right) \notin \nabla_{H_{1}+H_{2}} X_{1} \times X_{2}$. Thereby, $\nabla_{H_{1}+H_{2}} X_{1}$ $\times X_{2} \subset \nabla_{H_{1}} X_{1} \times \nabla_{H_{2}} X_{2}$.

REMARK. Theorem 1 can be obtained from Theorem 2. For we have

$$
\begin{aligned}
\mathrm{Cl}\left(\nabla_{H_{1}} X_{1}\right) \times \mathrm{Cl}\left(\nabla_{H_{2}} X_{2}\right) & =\mathrm{Cl}\left(\nabla_{H_{1}} X_{1} \times \nabla_{H_{2}} X_{2}\right) \\
& =\mathrm{Cl}\left(\nabla_{H_{1}+H_{2}} X_{1} \times X_{2}\right) .
\end{aligned}
$$

TheORem 3. If the Dirichlet problem for $X_{1} \times X_{2}$ relative to $H_{1}+H_{2}$ is solvable, then it is solvable for $X_{1}$ relative to $H_{1}$ and for $X_{2}$ relative to $H_{2}$.

Proof. We show that nonsolvability for either $X_{1}$ relative to $H_{1}$ or $X_{2}$ relative to $H_{2}$ implies nonsolvability for $X_{1} \times X_{2}$ relative to $H_{1}+H_{2}$. We will use the uniqueness of harmonic measures characterization of solvability of the Dirichlet problem. For definiteness we may assume that the Dirichlet problem is not solvable for $X_{1}$ relative to $H_{1}$. Then there exists $x_{0} \in X_{1}$ such that $M_{x_{0}{ }_{1}{ }_{1} H_{1}}\left(H_{1}\right) \supset\left\{u_{1}, u_{2}\right\}$ where $u_{1} \neq u_{2}$. The following argument is basically the same as the proof of $\nabla_{H_{1}+H_{2}} X_{1} \times X_{2} \subset \nabla_{H_{1}} X_{1} \times \nabla_{H_{2}} X_{2}$. The equality $\partial_{H_{1}} X_{1}$ $\times \partial_{H_{2}} X_{2}=\partial_{H_{1}+H_{2}} X_{1} \times X_{2}$ is crucial in order that the functions introduced be well-defined. Fix $y_{0} \in \partial_{H_{2}} X_{2}$. Define $\psi: C\left(\partial_{H_{1}+H_{2}} X_{1} \times X_{2}\right)$ $\rightarrow C\left(\partial_{H_{1}} X_{1}\right)$ as follows: if $\hat{f} \in C\left(\partial_{H_{1}+H_{2}} X_{1} \times X_{2}\right)$, then $\psi(\hat{f})(x)=\hat{f}\left(x, y_{0}\right)$ for all $x \in \partial_{H_{1}} X_{1}$. As in the proof of Theorem 7.2, $\psi$ is a positive linear map. Now define $\hat{u}_{1}, \hat{u}_{2}$ on $C\left(\partial_{H_{1}+H_{2}} X_{1} \times X_{2}\right)$ by $\hat{u}_{i}(\hat{f})=u_{i}(\psi(\hat{f}))$ for all $\hat{f} \in C\left(\partial_{H_{1}+H_{2}} X_{1} \times X_{2}\right), i=1,2$. Then $\hat{u}_{1}$ and $\hat{u}_{2}$ are positive linear functionals on $C\left(\partial_{H_{1}+H_{2}} X_{1} \times X_{2}\right)$. Let $\left[h_{1}+h_{2}\right] \in H_{1}+H_{2}$. Since $h_{1}+h_{2}\left(y_{0}\right)$ $\in H_{1}$ and $u_{i} \in M_{x_{0}}^{\partial H_{1} X_{1}}\left(H_{1}\right)$ for $i=1,2$, we have

$$
\begin{aligned}
\mathfrak{a}_{i}\left(\left[h_{1}+h_{2}\right] \mid \partial_{H_{1}+H_{2}} X_{1} \times X_{2}\right) & =u_{i}\left(h_{1}+h_{2}\left(y_{0}\right) \mid \partial_{H_{1}} X_{1}\right) \\
& =h_{1}\left(x_{0}\right)+h_{2}\left(y_{0}\right)=\left[h_{1}+h_{2}\right]\left(x_{0}, y_{0}\right) .
\end{aligned}
$$

Thus,

$$
u_{i} \in M_{\left(x_{0}, y_{0}\right)}^{\partial_{H_{1}+H_{2}} X_{1} \times X_{2}} \quad \text { for } i=1,2 .
$$

Now let $g \in C\left(\partial_{H_{1}} X_{1}\right)$ such that $u_{1}(g) \neq u_{2}(g)$. Define

$$
\hat{\mathrm{g}} \in C\left(\partial_{H_{1}+H_{2}} X_{1} \times X_{2}\right)
$$

by $\hat{g}(x, y)=g(x)$ for all $(x, y) \in \partial_{H_{1}+H_{2}} X_{1} \times X_{2}$. Then $\hat{u}_{1}(\hat{g})=u_{1}(\psi(\hat{g}))$ $=u_{1}(g)$ and $\mathfrak{u}_{2}(\hat{\mathrm{g}})=u_{2}(\psi(\hat{\mathrm{g}}))=u_{2}(g)$. Therefore, $\mathfrak{u}_{1} \neq \mathfrak{u}_{2}$. It follows that there is more than one $H_{1}+H_{2}$-harmonic measure belonging to the 
point $\left(x_{0}, y_{0}\right)$. Consequently, the Dirichlet problem is not solvable for $X_{1} \times X_{2}$ relative to $H_{1}+H_{2}$.

The next theorem is a partial converse of the already proved statement: nonsolvability of the Dirichlet problem for either $X_{1}$ relative to $H_{1}$ or $X_{2}$ relative to $H_{2}$ implies nonsolvability for $X_{1} \times X_{2}$ relative to $H_{1}+H_{2}$. Once again the equality $\partial_{H_{1}} X_{1} \times \partial_{H_{2}} X_{2}=\partial_{H_{1}+H_{2}} X_{1} \times X_{2}$ is crucial.

Theorem 4. Suppose there exists $\left(x_{0}, y_{0}\right) \in X_{1} \times \partial_{H_{2}} X_{2}$ such that

$$
M_{\left(x_{0}, y_{0}\right)}^{\partial_{H_{1}+H_{2} X_{1} \times X_{2}}} \supset\left\{\hat{u}_{1}, \hat{u}_{2}\right\}
$$

where $\mathfrak{a}_{1} \neq \mathfrak{u}_{2}$ and $S_{\hat{u}_{i}} \subset \partial_{H_{1}} X_{1} \times\left\{y_{0}\right\}$ for $i=1,2$, where $S_{\hat{u}_{i}}$ denotes the support of $\hat{u}_{i}$. (In particular, the Dirichlet problem is not solvable for $X_{1} \times X_{2}$ relative to $H_{1}+H_{2}$.) Then the Dirichlet problem is not solvable for $X_{1}$ relative to $H_{1}$.

Proof. Define $\rho: C\left(\partial_{H_{1}} X_{1}\right) \rightarrow C\left(\partial_{H_{1}+H_{2}} X_{1} \times X_{2}\right)$ as follows: if $f \in C\left(\partial_{H_{1}} X_{1}\right)$ then $\rho(f)(x, y)=f(x)$ for all $(x, y) \in \partial_{H_{1}+H_{2}} X_{1} \times X_{2}$. Clearly, $\rho(f) \in C\left(\partial_{H_{1}+H_{2}} X_{1} \times X_{2}\right)$ and $\rho$ is a positive preserving linear map. Now define $u_{1}, u_{2}$ on $C\left(\partial_{H_{1}} X_{1}\right)$ by $u_{i}(f)=a_{i}(\rho(f))$ for all $f \in C\left(\partial_{H_{1}} X_{1}\right)$ and $i=1,2$. Then $u_{1}$ and $u_{2}$ are positive linear functionals on $C\left(\partial_{H_{1}} X_{1}\right)$. Let $h \in H_{1}$. Since $[h+0] \in H_{1}+H_{2}$ and $a_{i} \in M_{\left(x_{0}, y_{0}\right)}^{\partial_{H_{2}}+H_{2} X_{1} \times X_{2}}$ for $i=1,2$, we have $u_{i}\left(h \mid \partial_{H_{1}} X_{1}\right)=u_{i}\left([h+0] \mid \partial_{H_{1}+H_{2}} X_{1} \times X_{2}\right)=h\left(x_{0}\right)$. Thus, $u_{i} \in M_{x_{0}}^{\partial_{H_{1}} X}$ for $i=1$, 2. Now let $\hat{g} \in C\left(\partial_{H_{1}+H_{2}} X_{1} \times X_{2}\right)$ such that $a_{1}(\hat{g}) \neq \mathfrak{a}_{2}(\hat{g})$. Define $g \in C\left(\partial_{H_{1}} X_{1}\right)$ by $g(x)=\hat{g}\left(x, y_{0}\right)$ for all $x \in \partial_{H_{1}} X_{1}$. Clearly, $g \in C\left(\partial_{H_{1}} X_{1}\right)$. Now $u_{i}(g)=a_{i}(\rho(g))$ for $i=1$, 2. But $\rho(g)(x, y)$ $=\hat{g}\left(x, y_{0}\right)$ for all $(x, y) \in \partial_{H_{1}+H_{2}} X_{1} \times X_{2}$. Thus, $\rho(g)\left|S_{\hat{u}_{i}}=\hat{g}\right| S_{\hat{u}_{i}}$ for $i=1$, 2 since $S_{\hat{u}_{i}} \subset \partial_{H_{1}} X_{1} \times\left\{y_{0}\right\}$. Hence, $a_{i}(\rho(g))=a_{i}(\hat{g})$ for $i=1,2$. Thus, $u_{1}(g) \neq u_{2}(g)$. Consequently, more than one $H_{1}$-harmonic measure belongs to the point $x_{0}$. Thus, the Dirichlet problem is not solvable for $X_{1}$ relative to $H_{1}$.

Of course, if there exists $\left(x_{0}, y_{0}\right) \in \partial_{H_{1}} X_{1} \times X_{2}$ such that

$$
M_{\left(x_{0}, y_{0}\right)}^{\partial_{H_{1}+H_{2} X_{1} \times X_{2}}} \supset\left\{a_{1}, a_{2}\right\}
$$

where $\mathfrak{u}_{1} \neq \mathfrak{a}_{2}$ and $S_{\hat{u}_{i}} \subset\left\{x_{0}\right\} \times \partial_{H_{2}} X_{2}$, then a similar proof shows that the Dirichlet problem is not solvable for $X_{2}$ relative to $H_{2}$.

\section{REFERENCES}

1. H. Bauer, Silooscher Rand und Dirichletsches Problem, Ann. Inst. Fourier (Grenoble) 11 (1961), 89-136.

2. E. Bishop and K. de Leeuw, The representation of linear functionals by measures on sets of extreme points, Ann. Inst. Fourier (Grenoble) 9 (1959), 305-331.

Rutgers, The State University 